\title{
Relationship between gait kinematics and walking energy expenditure during pregnancy in South African women
}

\author{
Zarko Krkeljas ${ }^{1,2^{*}}$ (D) and Sarah Johanna Moss ${ }^{1}$
}

\begin{abstract}
Background: Various musculoskeletal changes occurring during pregnancy may lead to the change in gait and contribute to the increase in walking energy expenditure. Previous research indicates that changes in gait mechanics may lead to the increase in mechanical work required during walking. However, there is little information to indicate if changes in gait mechanics during pregnancy have impact on active or total energy expenditure. Therefore, the primary aim of this study was to investigate the relationship between changes in gait kinematics and walking energy expenditure in pregnant women.

Methods: Thirty-five women (mean age $=27.5 \pm 6.1$ years) volunteered for the study during various stages of pregnancy (1st trimester average $=12.1 \pm 2.2$ weeks; 2 nd trimester $=22.3 \pm 2.6$ weeks; $3 r d$ trimester $=31.4 \pm 2.6$ weeks). 3D motion analysis was used to assess changes in kinematic parameters during walking at self-selected pace. Resting metabolic rate, and walking energy expenditure expressed in terms of rate and cost of $\mathrm{O}_{2}$ were analysed with portable metabolic analyser.

Results: Only medio-lateral deviation of centre of gravity (COGML) increased 13.6\% between the 1st and 2nd, and 39.3\% between 2 nd and $3 r d$ trimester $(p \leq 0.001)$. However, self-selected walking speed depicted strong significant positive linear relationship with net $\mathrm{O}_{2}$ rate $(r=0.70 ; p \leq 0.001)$, and was strongly associated with the vertical excursion of the $\operatorname{COG}(r=0.75, p \leq 0.001)$.

Conclusions: Changes in gait mechanics during pregnancy may lead to an increase in walking energy expenditure. However, the consequent increase in walking energy cost may not be sufficient to offset the natural energy sparing mechanism.
\end{abstract}

Keywords: Gait, Pregnancy, Energy expenditure, Centre of gravity, Kinematics

\section{Background}

Energy sparing during pregnancy is considered an inherent evolutionary biological mechanism [1]. There are numerous compensatory mechanisms that may be utilized to gain positive energy balance [2]. However, the energy required for foetal development is relatively small [3], and well-nourished mothers have adequate fat stores to provide for the additional energy needed for development. Although reducing the amount of walking or even reducing the walking speed are behavioural changes in

\footnotetext{
*Correspondence: zarkokrkeljas@gmail.com

'Physical Activity, Sport and Recreation Research Focus Area, North-West University, Private Bag x6001, Internal Box 481, Potchefstroom 2520, South Africa

${ }^{2}$ Duke Kunshan University, 8 Duke Avenue, Kunshan, Jiangsu Province 215316, China
}

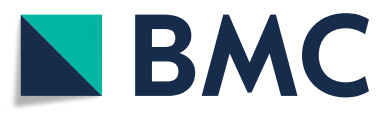

(c) The Author(s). 2018 Open Access This article is distributed under the terms of the Creative Commons Attribution 4.0 International License (http://creativecommons.org/licenses/by/4.0/), which permits unrestricted use, distribution, and reproduction in any medium, provided you give appropriate credit to the original author(s) and the source, provide a link to the Creative Commons license, and indicate if changes were made. The Creative Commons Public Domain Dedication waiver (http://creativecommons.org/publicdomain/zero/1.0/) applies to the data made available in this article, unless otherwise stated. chanics during pregnancy may lead to an increase in walking energy expenditure [4]. Yet, it is not clear if the relative energy changes as a result of alterations in gait mechanics have a significant impact on overall energy balance during pregnancy.

The change in trunk moment of inertia during pregnancy causes various compensations and adaptions in posture and gait mechanics that may result in the increase in walking energy expenditure [4]. As a significant portion of the metabolic cost of walking is attributed to the work required to move the body's centre of mass $(\mathrm{COM})[5,6]$, any changes in gait kinematics affecting path of the $\mathrm{COM}$ would reflect on the energy expenditure [4]. It is well 
established that changes in gait kinematics are associated with changes in primary gait determinants $[7,8]$ which may lead to increase in metabolic cost of locomotion, yet there is a lack of studies investigating this relationship during pregnancy.

For example, self-selected walking speed decreases later in pregnancy $[2,9]$, which is associated with smaller trunk rotations [10], and consequent "flattening" of the $\mathrm{COM}$ which is associated with the decrease in walking metabolic cost. Additionally, step width increases during pregnancy which is associated with promotion of balance during walking $[9,11]$, but results in greater side-to-side movements of the COM leading to greater mechanical work and consequent increase in walking energy expenditure. Similarly, a decrease in stride length, an increase in double support time, and a decrease in step frequency have also been noted during pregnancy [9], and may be associated with changes in movement of the COM during walking. In addition, anterior weight distribution places an increased demand on the lumbar spine and the abdominal muscles, causing an anterior pelvic tilt and consequently lumbar lordosis commonly reported in pregnancy [12]. Postural adaptations will lead to anterior-posterior changes in COM [13].

On the other hand, changes in active and total energy expenditure during pregnancy may be interpreted through quality or quantity of movement. For example, since the net daily energy expenditure during pregnancy does not differ significantly from pre-pregnancy for the same activity $[2,3,14-16]$, this indicates either a decrease in the pace of performing that activity [16], or an effective mechanical adaptation in the execution of a physical activity [2]. In addition, an increase in resting metabolic rate (RMR) during gestation and a simultaneous decrease in daily net oxygen consumption $\left(\mathrm{VO}_{2}\right)$ may also be an indication of a strategy for a more economical movement $[2,16]$.

While various gait parameters have been investigated during pregnancy, only walking speed has been investigated relative to the energy expenditure [2]. Therefore, the primary aim of this study is to investigate the relationship between the gait kinematics and the metabolic cost of walking during pregnancy.

\section{Methods}

This study was derived from a larger Habitual Activity Patterns during Pregnancy (HAPPY) study that investigated the changes in objectively determined physical activity patterns during pregnancy and their influence on various pregnancy outcomes. Thirty-five pregnant women at different stages of pregnancy, mean age 27.5 years $($ S.D. $=6.1)$, were recruited by advertisements in the local press, the consulting rooms of local gynaecologists, and a local health clinic in Potchefstroom,
North West Province, South Africa. To participate in the study, women had to be healthy, between the ages of 18 and 40 years, without mental or physical disability, able to complete the test protocol, and not be considered a high-risk pregnancy according the guidelines of the American College of Sports Medicine (ACSM) [17]. Participants were allowed to return for additional measures at different stages of pregnancy. The women gave written consent for participation before data collection. A translator was available in the case of language barriers. The study was approved by the Human Research Ethics committee of North-West University (NWU-0044-10-A1).

Procedures for walking and resting energy expenditure, and gait analysis were previously described in Krkeljas and Moss [4], hence only a brief description of the methodology will be provided in the following section.

To measure RMR participants lay still for $5 \mathrm{~min}$ on their left side to ensure a resting state, after which Fitmate metabolic system (Cosmed Fitmate, Italy) was attached. RMR gas exchange was monitored for $16 \mathrm{~min}$ per Fitmate RMR protocol.

Walking energy expenditure was measured using the portable $\mathrm{K}_{4} \mathrm{~b}^{2}$ (Cosmed, Italy) metabolic system, while participants walked at a self-selected pace along a 30-m-long oval track in the laboratory until steady state was reached. Steady state was considered by heart rate variation being no more than \pm 3 beats per minute (bpm), and less than $5 \%$ variation in respiratory quotient (RQ) [18], during which RQ of less than $\leq 0.99$ had to be maintained [19]. The following parameters were recorded: walking volume of oxygen $\left(\mathrm{VO}_{2}\right)(\mathrm{ml} / \mathrm{kg} / \mathrm{min})$, $\mathrm{RQ}, \mathrm{RMR}$ (kcal/day), heart rate (bpm).

Full body 3D gait analysis was completed using eight Oqus 300+ cameras from Qualisys Motion Analysis System (Qualisys, Sweden) and collected at $220 \mathrm{~Hz}$. Reflective markers were placed according to CAST/IK/HH (calibrated anatomical systems technique/Helen-Heyes/ inverse kinematics) gait model. During dynamic trials, participants were instructed to walk in a straight line at a self-selected pace along a $15 \mathrm{~m}$ laboratory walkway embedded with four AMTI BP400600 force plates (AMTI, Watertown, MA, USA). Motion and ground reaction force data were collected simultaneously for $5 \mathrm{~s}$ in the middle part of the runway. Only trials in which the participant's foot landed entirely on a force plate for three consecutive steps (i.e. at full stride), were considered for inclusion in the data set. The subjects continued walking until three trials at full stride were completed. The participants were instructed to stop and rest as long as necessary, should they have felt tired at any stage of the examination of their gait. None did so.

\section{Data analysis}

Gait kinematics analysed were: walking speed (time it takes to complete a single stride measured in $\mathrm{m} / \mathrm{s}$ ), step 
length (distance between reflective markers placed on Achilles tendon measured in meters) and step width (distance between left and right foot joint centres determined as the midpoint between lateral and medial malleoli measured in meters) normalized for leg length, double-support time (time from heel strike to push of the opposite foot measured in seconds), vertical and medio-lateral excursion of centre of gravity $(\mathrm{COG})(\mathrm{m})$. The vertical force of $5 \%$ of body weight was used as a threshold for heel contact and toe-off.

During walking trials, the data were inspected for gaps in marker trajectories. The default gap-fill function was applied for gaps of no more than 10 frames using non-uniform rational basis spline (NURB) spline interpolation. No walking data trials analysed had gaps of more than 10 frames. Once the walking trials were limited to include only completed strides, the data were exported to Visual 3D-motion analysis software (C-Motion, MD, USA) for processing. The kinematic parameters were low-pass filtered with a bidirectional Butterworth filter with a $10 \mathrm{~Hz}$ cut-off frequency to remove noise from the differentiation process with zero-phase distortion [20].

Metabolic energy expenditure was reported as $\mathrm{O}_{2}$ consumption $\left(\mathrm{O}_{2}\right.$ rate $)(\mathrm{ml} / \mathrm{kg} / \mathrm{min})$, and to demonstrate the physiological work $\left(\mathrm{O}_{2}\right.$ cost $)$ for a given task by normalizing the $\mathrm{O}_{2}$ consumption for speed $(\mathrm{ml} / \mathrm{kg} / \mathrm{m})$ [21]. In addition, to reduce the impact of changes in RMR $\mathrm{O}_{2}$ consumption was also analysed as net energy consumption by deducing the RMR from total energy expenditure. The net $\mathrm{O}_{2}$ cost may also be less sensitive to changes in walking speed [22]. This method in principle accounts for pregnancy-induced changes; however, nothing in the literature was found that addressed this normalization process in respect of gait in pregnancy.

\section{Statistical analysis}

Data are presented as means \pm standard deviation as specified. Shapiro-Wilks test was used to assess the data distribution. Levene's test was used to determine whether there were any differences in variances between trimesters. A one-way ANOVA was used to assess differences between trimesters for the women's physical characteristics, gait kinematics, and gait metabolic energy expenditure. LSD post hoc correction was set at $\alpha=0.05$ for all analyses. ANOVA was confirmed via a KruskalWallis test for non-parametric data. If there were significant differences in variances between trimesters, Games-Howell post-hoc test was conducted. Pearson's product correlations were computed to determine correlations between outcome variables. Therefore, the trimester of pregnancy is considered an independent variable, while kinematic and metabolic data are the dependent variables. All analyses were performed using SPSS v.21.0 (IBM Corp., Armonk, NY).

\section{Results}

Participants' descriptive parameters in respect of anthropometrics, gait kinematics, and energy expenditure per trimester are depicted in Table 1.

Coefficient of variation in first trimester for all metabolic and kinematic variables ranged from $6.4 \%$ for walking speed to $35.2 \%$ for net walking energy expenditure. Weight gain per trimester was within the range recommended by the Institute of Medicine (6.7-11.2 kg) [23]. Based on self-reported pre-pregnancy weight, participants were on average borderline overweight with a mean of $25.1 \pm 5.5 \mathrm{~kg} / \mathrm{m}^{2}$ [23].

Differences between the trimesters in gross and net walking energy expenditure were not significant. Similarly, while absolute REE was greater in each consequent trimester, the differences were not statistically significant. When normalized for the mass gain REE was decreasing, although these differences were also not statistically significant. The mean respiratory quotient $(\mathrm{RQ})$ remained below 1.0 (mean $=0.91 \pm 0.07)$ an indication of aerobic metabolism dominant throughout pregnancy $[21,24]$. However, the RQ was significantly higher in the 3rd trimester $(p \leq 0.05)$ and was close to $1.0(0.96 \pm 0.02)$, which signifies a potential change in metabolic process.

Relative to gait kinematics, only $\mathrm{COG}_{\mathrm{ML}}$ significantly increased between trimesters $(p \leq 0.001)$, while walking speed, step length, and step width remained unchanged (Table 1). Changes in gait kinematics, step width and $\mathrm{COG}_{\mathrm{ML}}$ were associated with mass gain rather than the absolute mass $(r=0.38, p \leq 0.01$ and $r=0.50, p \leq 0.001$ respectively) (Table 2 ), whereas changes in walking speed were inversely related to the mass $(r=-0.43$, $p \leq 0.001)$. However, relative to the net energy rate and cost, only self-selected walking speed $(r=0.70, r=0.53$, $p \leq 0.001$, respectively) and COGv $(r=0.45, p \leq 0.01$ and $r=0.30, p \leq 0.05)$ showed significant association.

Net walking energy cost and rate are significantly associated with walking speed (Figs. 1 and 2, respectively), whereas gross energy expenditure shows weak and non-significant relationship.

\section{Discussion}

The principal findings of this study are two-fold: firstly, self-selected walking speed has strong significant relationship with net walking energy expenditure during pregnancy; and secondly, the relative mass gain, rather than the absolute mass is a primary factor associated with changes in gait mechanics which may lead to increase in walking energy expenditure. 
Table 1 Participants' characteristics with respect to gait kinematics and walking energy expenditure per trimester

\begin{tabular}{|c|c|c|c|c|}
\hline Measure & $\begin{array}{l}\text { 1st trim. } \\
\text { Mean } \pm S D\end{array}$ & $\begin{array}{l}\text { 2nd trim. } \\
\text { Mean } \pm S D\end{array}$ & $\begin{array}{l}\text { 3rd trim. } \\
\text { Mean } \pm S D\end{array}$ & Sig. $(p)$ \\
\hline Participants (n) $^{z}$ & 14 & 20 & 10 & \\
\hline Age (years) & $28.1 \pm 5.5$ & $27.1 \pm 6.1$ & $26.6 \pm 6.6$ & 0.83 \\
\hline Gestation (wks) & $12.1 \pm 2.2$ & $22.3 \pm 2.6$ & $31.4 \pm 2.6$ & - \\
\hline Height $(\mathrm{cm})$ & $160.8 \pm 5.9$ & $160.2 \pm 6.8$ & $161.4 \pm 7.2$ & 0.89 \\
\hline Mass $(\mathrm{kg})$ & $62.7 \pm 10.5$ & $71.3 \pm 16.6$ & $78.8 \pm 14.7$ & 0.08 \\
\hline$B M I_{\left(\mathrm{kg} / \mathrm{m}^{2}\right)}$ & $24.3 \pm 4.0$ & $27.7 \pm 6.2$ & $29.9 \pm 4.9$ & 0.08 \\
\hline$M_{\text {gain (kg) }}$ & $1.1 \pm 3.1^{a, c}$ & $5.3 \pm 2.8^{b, c}$ & $13.8 \pm 7.9^{a, b}$ & $0.00^{* * *}$ \\
\hline $\mathrm{S}_{(\mathrm{m} / \mathrm{s})}$ & $1.09 \pm 0.07$ & $1.10 \pm 0.11$ & $1.01 \pm 0.19$ & 0.16 \\
\hline Stride length ${ }^{*}$ & $0.69 \pm 0.06$ & $0.72 \pm 0.06$ & $0.70 \pm 0.05$ & 0.17 \\
\hline Step width ${ }^{*}$ & $0.06 \pm 0.02$ & $0.07 \pm 0.02$ & $0.07 \pm 0.02$ & 0.30 \\
\hline DS time $(\mathrm{s})$ & $0.12 \pm 0.03$ & $0.11 \pm 0.03$ & $0.13 \pm 0.06$ & 0.35 \\
\hline $\mathrm{COG}_{V(\mathrm{~cm})}$ & $3.37 \pm 0.56$ & $3.55 \pm 0.73$ & $3.22 \pm 0.726$ & 0.53 \\
\hline $\operatorname{COG}_{M L}(\mathrm{~cm})$ & $2.06 \pm 0.42^{\mathrm{a}}$ & $2.34 \pm 0.89^{b}$ & $3.26 \pm 0.57^{a, b}$ & $0.001^{* *}$ \\
\hline REE (kcal/day) & $1405.7 \pm 183.7$ & $1488.1 \pm 190.0$ & $1578.0 \pm 216.1$ & 0.12 \\
\hline $\operatorname{REE}_{(\mathrm{kcal} / \mathrm{kg} / \mathrm{day})}$ & $22.7 \pm 2.6$ & $21.4 \pm 2.5$ & $20.9 \pm 2.2$ & 0.40 \\
\hline Gross $\mathrm{O}_{2}(\mathrm{ml} / \mathrm{kg} / \mathrm{min})$ & $10.93 \pm 2.46$ & $9.66 \pm 1.45$ & $10.39 \pm 2.01$ & 0.26 \\
\hline Gross $\mathrm{O}_{2}(\mathrm{~m} / \mathrm{kg} / \mathrm{m})$ & $0.17 \pm 0.04$ & $0.15 \pm 0.02$ & $0.17 \pm 0.03$ & 0.16 \\
\hline Net $\mathrm{O}_{2}(\mathrm{~m} / / \mathrm{kg} / \mathrm{min})$ & $9.15 \pm 3.26$ & $8.04 \pm 2.72$ & $8.51 \pm 3.69$ & 0.60 \\
\hline Net $\mathrm{O}_{2(\mathrm{~m} / \mathrm{kg} / \mathrm{m})}$ & $0.12 \pm 0.04$ & $0.10 \pm 0.02$ & $0.12 \pm 0.02$ & 0.16 \\
\hline $\mathrm{RQ}$ & $0.90 \pm 0.11^{b}$ & $0.89 \pm 0.06^{a}$ & $0.96 \pm 0.02^{a, b}$ & $0.04^{*}$ \\
\hline
\end{tabular}

$\overline{a, b, c}$ denotes significance between respective trimesters; $M_{\text {gain }}=$ mass gain from pre-pregnancy (i.e. total mass gain); $S=$ walking speed; $D S=$ double support; $\mathrm{COGV}=$ vertical excursion of the centre of gravity; $\mathrm{COG}_{\mathrm{ML}}=$ medio-lateral centre of gravity displacement; ${ }^{*}=$ normalized for leg length; $\mathrm{O}_{2}=$ walking volume of oxygen; $\mathrm{RQ}=$ respiratory quotient; Net $\mathrm{O}_{2}=$ energy expenditure only necessary for walking $\left(\mathrm{TEE}_{\text {gait }}-\mathrm{REE}\right)$; trim. $=$ trimester; ${ }^{\mathrm{z}}$ Several participants were measured in multiple stages

In this study, although the differences between trimesters in gait kinematics and walking energy expenditure were not statistically significant, there were significant associations between gait kinematics and walking energy expenditure. Similarly to previous studies, self-selected walking speed during third trimester was lower than the first or second trimesters [2,9], although in this study this decrease was not statistically significant. Changes between trimesters in gross and net walking energy expenditures were also not significantly different. However, self-selected walking speed showed strong significant relationship with net walking energy rate $(\mathrm{ml} / \mathrm{kg} / \mathrm{min})$ and walking economy $(\mathrm{ml} / \mathrm{kg} / \mathrm{m})$ (Figs. 1 and 2, respectively), while there was a lack of association with the gross

Table 2 Pearson correlations between body weight, gait kinematics and walking energy expenditure

\begin{tabular}{|c|c|c|c|c|c|c|c|}
\hline & $\mathrm{COG}_{V(\mathrm{~m})}$ & $\mathrm{COG}_{\mathrm{ML}(\mathrm{m})}$ & $\mathrm{GR} \mathrm{O}_{2(\mathrm{~m} / \mathrm{kg} / \mathrm{min})}$ & $\begin{array}{l}\mathrm{NR} \mathrm{O}_{2} \\
(\mathrm{ml} / \mathrm{kg} / \mathrm{min})\end{array}$ & $\begin{array}{l}\mathrm{NC} \mathrm{O}_{2} \\
(\mathrm{ml} / \mathrm{kg} / \mathrm{m})\end{array}$ & Mass $_{(\mathrm{kg})}$ & $\begin{array}{l}M_{\text {gain }} \\
(\mathrm{kg}) \\
\end{array}$ \\
\hline Mass $_{(\mathrm{kg})}$ & -0.18 & 0.24 & -0.15 & -0.19 & -0.11 & - & - \\
\hline$M_{\text {gain }(\mathrm{kg})}$ & -0.01 & $0.50^{* * *}$ & -0.04 & -0.08 & -0.02 & $0.43^{* *}$ & - \\
\hline $\mathrm{S}(\mathrm{m} / \mathrm{s})$ & $0.75^{* * *}$ & -0.18 & $0.30^{*}$ & $0.70^{* * *}$ & $0.53^{* * *}$ & $-0.43^{* *}$ & -0.27 \\
\hline Stride length ${ }^{a}$ & $0.32^{*}$ & $-0.39^{* *}$ & -0.18 & 0.09 & -0.01 & -0.01 & -0.29 \\
\hline Step width ${ }^{a}$ & 0.09 & -0.02 & 0.20 & 0.19 & 0.22 & 0.05 & $0.38^{* *}$ \\
\hline DS time ${ }_{(\mathrm{s})}$ & -0.17 & 0.00 & 0.23 & -0.23 & -0.18 & 0.15 & $0.34^{*}$ \\
\hline $\operatorname{COG}_{V(m)}$ & - & - & 0.11 & $0.45^{* *}$ & $0.30^{*}$ & -0.18 & -0.01 \\
\hline $\operatorname{COG}_{M L}(\mathrm{~m})$ & -0.03 & - & -0.10 & -0.12 & -0.09 & 0.24 & $0.50^{* * *}$ \\
\hline $\operatorname{REE}_{(\mathrm{kcal} / \mathrm{day} / \mathrm{kg})}$ & - & - & 0.04 & 0.03 & -0.04 & $-0.85^{* * *}$ & $-0.31^{*}$ \\
\hline
\end{tabular}

${ }^{*} p \leq 0.05 ;{ }^{* *} \leq 0.01 ;{ }^{* * *} \leq 0.001 ; \mathrm{M}_{\text {gain }}=$ mass relative to pre-pregnancy mass; $\mathrm{S}=$ walking speed; $\mathrm{DS}=$ double support; COGv = vertical excursion of the centre of gravity; $\mathrm{COG}_{\mathrm{ML}}=$ medio-lateral centre of gravity displacement; ${ }^{\mathrm{a}}$ normalized for leg length; $\mathrm{GR}_{2}=$ gross $\mathrm{O}_{2}$ rate; $\mathrm{NR} \mathrm{O}_{2}=$ net $\mathrm{O}_{2}$ rate; $\mathrm{NC} \mathrm{O}_{2}=$ net $\mathrm{O}_{2}$ cost; $\mathrm{REE}=$ resting energy expenditure 


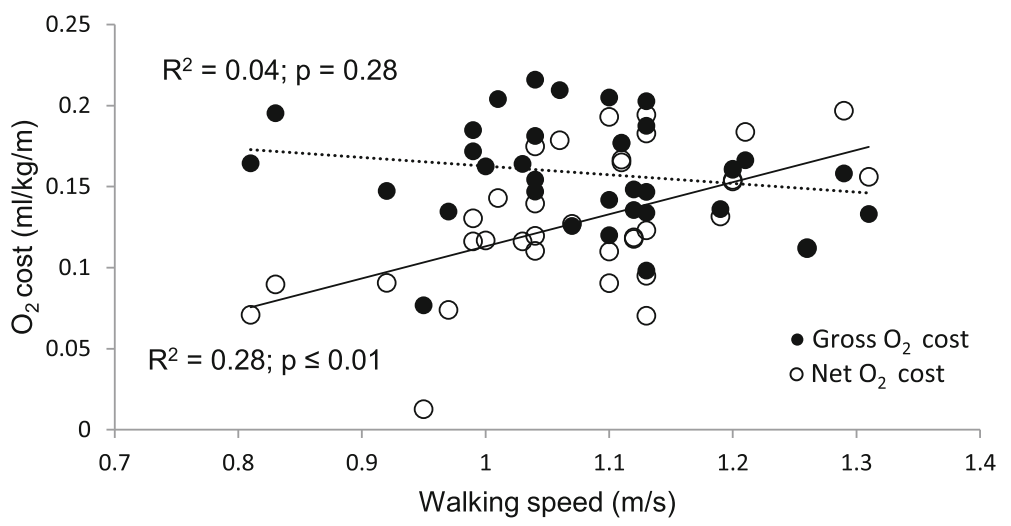

Fig. 1 Gross and net energy cost relative to walking speed during pregnancy

energy expenditure. Since gross energy expenditure contains REE, the variability in REE which is associated with physiological changes due to foetal development, would not be related to the energy expenditure required for walking. Increase in resting energy expenditure (kcal/ day) (Table 1) is associated with the increase in mass (Table 2), although the lack of statistical differences may be attributed to the large variability in mass gain between the participants, or the differences in self-reported pre-pregnancy weight.

The relationship of speed of walking and net energy expenditure is largely determined by the $\mathrm{COG}_{\mathrm{v}}(r=0.70$, $p \leq 0.001 ; r=0.45, p \leq 0.01$, respectively) (Table 2). Given that the motion of the COG may be regarded as the summation of all forces that act on the body, the significant portion of the total metabolic cost during walking should be attributed to the work required to move the COG $[5,6]$, especially as the weight of the body increases as in pregnancy. This effect has been demonstrated in our previous article [4]. This relationship indicates that the ability to increase walking efficiency is related to the principle of conservation of mechanical energy during walking that is maximized at certain speeds $[4,5,25]$, which participants in this study did not reach. The average self-selected walking speed of $1.08 \pm$ $0.11 \mathrm{~m} / \mathrm{s}$ did not significantly change during pregnancy and falls within previously reported range from $0.83 \mathrm{~m} / \mathrm{s}$ [10] to $1.5 \mathrm{~m} / \mathrm{s}$ [2].

While the changes in walking speed were associated with the absolute mass $(r=-0.43, p \leq 0.01)$, gait parameters associated with the greater stability during walking, step width and the time spent in double-support stage, were associated with the relative mass gain $(r=0.38$, $p \leq 0.01$ and $r=0.34, p \leq 0.05$, respectively). Due to weight distribution during pregnancy, the trunk moment of inertia increases leading to need for greater stability [20]. More stability during walking may be obtained by increasing double-support time, increase the step width, or both, in order to create a larger base of support. In addition, lower walking speeds results in an increased double support time, which gives pregnant women more time to react and control additional balance demands during walking $[9,20,26]$.

However, these gait changes may result in mechanically inefficient gait which may lead to increase in total energy expenditure [4]. Walking with the bigger base of support results in large side-to-side excursions of the centre of gravity (COG) [26], which may increase the

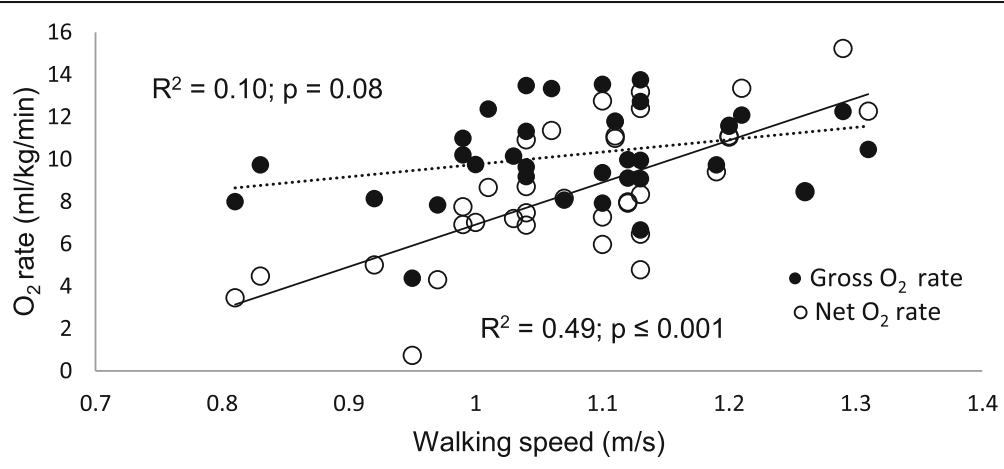

Fig. 2 Changes in gross and net energy rate relative to changes in walking speed during pregnancy 
energy demand as discussed earlier. The results in this study show $13.6 \%$ increase of medio-lateral excursions of centre of gravity $\left(\mathrm{COG}_{\mathrm{ML}}\right)$ between the first and second trimester, and $39.3 \%$ between second and third trimester $(p \leq 0.001)$. These changes were significantly related to relative mass gain $(r=0.50, p \leq 0.001)$, rather than the absolute mass. In late pregnancy, due to large mass gain, width of the pelvic girdle also increases in order to accommodate the growing foetus, which also leads to the increase in the width of the base of support [11] and consequently the increase in the step width during pregnancy [27].

While changes in gait mechanics may have a significant impact on walking energy expenditure, the metabolic cost of walking may not be sufficient to alter the overall net energy balance. The increase in absolute REE between the trimesters (although not statistically significant) was largely associated with the mass $(r=0.86$, $p \leq 0.001$ ), however, once normalized for the mass REE decreased between subsequent trimesters and showed strong negative correlation with the mass $(r=-0.85$, $p \leq 0.001)$, which is suggestive of energy conservation process during pregnancy associated with the changes in metabolism [2]. However, the difference in REE between the $1 \mathrm{st}$ and $3 \mathrm{rd}$ trimester was $1.8 \mathrm{kcal} / \mathrm{kg}$, indicating that energy sparing process in a woman with approximate weight of $65 \mathrm{~kg}$ (average pre-pregnancy weight in this study $=64.4 \pm 14.7 \mathrm{~kg}$ ), would conserve $117 \mathrm{kcal} /$ day only a 6.5 to $5.9 \%$ increase from 1800 to $2000 \mathrm{kcal} /$ day recommended daily caloric intake for healthy women of the same group and activity level as reported in this study. Considering the relationship of walking speed and net energy expenditure in this study, and the decrease from 1st to 3rd trimesters in walking speed, the difference in energy expenditure conservation by means of walking would equal to $0.5 \mathrm{kcal} / \mathrm{min}$ for the same individual. Therefore, for conservation of energy from changes in gait to have a meaningful impact on overall energy expenditure during pregnancy, women would have to walk continually for several hours.

The small impact changes in gait mechanics have on total energy expenditure, allows for gait mechanics to be altered for reasons such as balance or comfort, which may lead to mechanically inefficient gait [4], but without the significant impact on overall energy expenditure, which helps maintain overall net positive energy balance during pregnancy. Because pregnancy is characterized by the bearing of an extra and "valuable" load, and as such walking efficiency has to be combined with safety. While the additional burden of the growing fetus may increase the demand of mechanical energy, women tend to adopt a strategy that helps them maintain the rate of energy expenditure at a level that can be sustained for a relatively long time. This is also a strategy adopted by individuals who walk with a pathological condition [28]. Considering that the pre-pregnancy physical and physiological characteristics differ among the women studied, this is also the most likely source of large inter-subject variability in gait parameters during pregnancy reported across all similar studies.

The results of this study have to be considered in regard to the limitations presented during data collection. Firstly, not all the pre-pregnancy weight was obtained from participants' records and was therefore self-reported, which is known to be under-estimated at the times. Secondly, large withdrawal rates prevented longitudinal tracking, which would allow identification of the most common changes occurring during pregnancy in the parameters investigated.

\section{Conclusion}

The changes in gait mechanics during pregnancy may occur as a result of various adaptations and needs of the mother. It is likely that those changes will result in change in energy expenditure during walking. However, considering the inherent energy conservation process occurring during pregnancy, the changes in energy expenditure due to gait are not sufficient to significantly alter the overall positive energy balance.

\begin{abstract}
Abbreviations
BOS: Base of support; COG: Centre of gravity; $\mathrm{COG}_{\mathrm{ML}}$ : Medio-lateral excursions of the centre of gravity; COM: Centre of mass; GR: Gross rate; NC: Net cost; NR: Net rate; $\mathrm{O}_{2}$ : Oxygen (molecular formula); PA: Physical activity; REE: Resting energy expenditure; RMR: Resting metabolic rate; $\mathrm{RQ}$ : Respiratory quotient; TEE: Total energy expenditure; $\mathrm{VO}_{2}$ : Volume of oxygen consumption
\end{abstract}

\section{Acknowledgements}

We would like to acknowledge our co-worker, Abie van Oort, for assisting with the recruitment of the participants. In addition, I would like to extend our gratitude to the participants of the HAPPY-study and the clinic staff for assisting and supporting the recruitment of participants and translation when needed. I also convey our gratitude to The South African Sugar Association and the support of the Swiss South African Joint Research Programme.

\section{Funding}

The South African Sugar Association and National Research Foundation of South Africa funded the HAPPY project, however, funding was specific for the collection of physical activity data and resting metabolic rate.

\section{Availability of data and materials}

The datasets used and/or analysed during the current study are available from the corresponding author on reasonable request.

\section{Authors' contributions}

ZK carried out the walking energy expenditure study, designed the protocol, collected data, and drafted the manuscript. SJM is the principle investigator of the larger conceptual project, the HAPPY-study and participated in critically revising the manuscript, and gave the final approval for the version to be published. All authors read and approved the final manuscript.

\section{Ethics approval and consent to participate}

The study was approved by the Human Research Ethics committee of NorthWest University (NWU-0044-10-A1). Participants were asked to read and sign the informed consent if they agree to participate in the study. 


\section{Consent for publication}

Consent for publication of anonymous personal and testing data was obtained as part of the informed consent for the study.

\section{Competing interests}

The authors declare that they have no competing interests.

\section{Publisher's Note}

Springer Nature remains neutral with regard to jurisdictional claims in published maps and institutional affiliations.

Received: 12 February 2018 Accepted: 7 June 2018

Published online: 19 June 2018

\section{References}

1. Prentice AM, Goldberg GR, Davies HL, Murgatroyd PR, Scott W. Energysparing adaptations in human pregnancy assessed by whole-body calorimetry. Br J Nutr. 1989;62:5-22.

2. Byrne NM, Groves AM, McIntyre HD, Callaway LK. Changes in resting and walking energy expenditure and walking speed during pregnancy in obese women. Am J Nutr. 2011;94:819-30.

3. Melzer K, Schutz Y, Boulvain M, Kayser B. Pregnancy-related changes in activity energy expenditure and resting metabolic rate in Switzerland. Eur J Clin Nutr. 2009:63:1185-91.

4. Krkeljas Z, Moss SJ. Correlating mechanical work with energy consumption during gait throughout pregnancy. BMC Pregnancy Childbirth. 2015:15:303.

5. Bennett $B C$, Abel MF, Wolovick A, Franklin T, Allaire PE, Kerrigan DC, et al. Center of mass movement and energy transfer during walking in children with cerebral palsy. Arch Phys Med Rehabil. 2005;86:2189-94.

6. Donelan JM, Kram R, Kuo AD. Mechanical work for step-to-step transitions is a major determinant of the metabolic cost of human walking. J Exp Bio. 2002;205:3717-27.

7. Saunders $\mathrm{M}$, Inman VT. The major determinants in normal and pathological gait. J Bone Joint Surg. 1953;35-A:543-58.

8. Della Croce U, Riley PO, Lelas JL, Kerrigan DC, Della U, Riley PO, et al. A refined view of the determinants of gait. Gait Posture. 2001;14:79-84.

9. Carpes FP, Griebeler D, Kleinpaul JF, Mann L, Mota CB. Women able-bodied gait kinematics during and post pregnancy period. Bras J Biomech. 2008;9:33-40.

10. Wu WH, Meijer OG, Bruijn SM, Hu H, van Dieën JH, Lamoth CJC, et al. Gait in pregnancy-related pelvic girdle pain: amplitudes, timing, and coordination of horizontal trunk rotations. Eur Spine J. 2008;17:1160-9.

11. Foti T, Davids JR, Bagley A. A biomechanical analysis of gait during pregnancy. J Bone Joint Surg. 2000;82-A:625-32.

12. Ponnapula P, Boberg JS. Lower extremity changes experienced during pregnancy. J Foot Ankle Surg. 2010;49:452-8.

13. Krkeljas Z. Changes in gait and posture as factors of dynamic stability during walking in pregnancy. Hum Mov Sci. 2017;58:315-20.

14. Forsum E, Kabir N, Sudarskis A, Westeterp K. Total energy expenditure of healthy Swedish women during pregnancy and lactation. Am J Clin Nutr. 1992:56:334-42.

15. Lof M, Forsum E. Activity pattern and energy expenditure due to physical activity before and during pregnancy in healthy Swedish women. Br I Nutr. 2006;95(2):296-302.

16. Van Raaij JM, Schonk C, Vermaat-Miedema SH, Peek ME, Hautvast JG. Energy before, cost of walking at a fixed pace during, and after pregnancy. Am J Clin Nutr. 1990;51:158-61.

17. Artal R, OToole M. Guidelines of the American College of Obstetricians and Gynecologists for exercise during pregnancy and the postpartum period. Br J Sports Med. 2003;37:6-12

18. Thomas SS, Buckon CE, Schwartz MH, Sussman MD, Aiona MD. Walking energy expenditure in able-bodied individuals: a comparison of common measures of energy efficiency. Gait Posture. 2009;29:592-6.

19. Schutz Y. Dietary fat, lipogenesis and energy balance. Physiol Behav. 2004; 83:557-64.

20. Wu W, Meijer OG, Lamoth CJC, Uegaki K, van Dieën JH, Wuisman PIJM, et al. Gait coordination in pregnancy: transverse pelvic and thoracic rotations and their relative phase. Clin Biomech (Bristol, Avon). 2004;19:480-8.

21. Waters RL, Mulroy $\mathrm{S}$. The energy expenditure of normal and pathologic gait. Gait Posture. 1999;9:207-31.

22. Baker R, Hausch A, Mcdowell B. Reducing the variability of oxygen consumption measurements. Gait Posture. 2001;13:202-9.
23. Institute of medicine. Weight gain during pregnancy: Reexamining the guidelines. 2009. Accessed 2 Dec 2016.

24. Melzer K, Kayser B, Schutz Y. Respiratory quotient evolution during normal pregnancy: what nutritional or clinical information can we get out of it? Eur J Obstet Gynecol Reprod Biol. 2014;176:5-9.

25. Willems PA, Cavagna GA, Heglund NC. External, internal and total work in human locomotion. J Exp Biol. 1995;198:379-93.

26. Lymbery JK, Gilleard $\mathrm{W}$. The stance phase of walking during late pregnancy temporospatial and ground reaction force variables. J Am Podiatr Med Assoc. 2005;95:247-53.

27. Jang J, Hsiao KT, Hsiao-Wecksler ET. Balance (perceived and actual) and preferred stance width during pregnancy. Clin Biomech (Bristol, Avon). 2008;23:468-76.

28. Detrembleur C, Dierick F, Stoquart G, Chantraine F, Lejeune T. Energy cost, mechanical work, and efficiency of hemiparetic walking. Gait Posture. 2003;18:47-55

\section{Ready to submit your research? Choose BMC and benefit from:}

- fast, convenient online submission

- thorough peer review by experienced researchers in your field

- rapid publication on acceptance

- support for research data, including large and complex data types

- gold Open Access which fosters wider collaboration and increased citations

- maximum visibility for your research: over $100 \mathrm{M}$ website views per year

At BMC, research is always in progress.

Learn more biomedcentral.com/submissions 